\title{
Families of smooth curves on surface singularities and wedges
}

\author{
by GÉrard GonZALEZ-SPRINBERG and \\ Monique LeJeune-Jalabert (Grenoble)
}

\begin{abstract}
Following the study of the arc structure of singularities, initiated by J. Nash, we give criteria for the existence of smooth curves on a surface singularity $(S, O)$ and of smooth branches of its generic hypersurface section. The main applications are the following: the existence of a natural partition of the set of smooth curves on $(S, O)$ into families, a description of each of them by means of chains of infinitely near points and their associated maximal cycle and the existence of smooth curves on any sandwiched surface singularity. A wedge centered at a smooth curve on $(S, O)$ is essentially a one-parameter deformation of the parametrization of the curve. We show that there is no wedge centered at smooth curves of two different families.
\end{abstract}

Introduction. In this paper we investigate the infinitesimal geometry of the set of smooth curves on a surface singularity. Our motivation comes from a preprint $\left({ }^{1}\right)$ by J. Nash in which he initiates the study of the set of germs of parametrized curves, arcs in his terminology, on an algebraic or analytic variety over $\mathbb{C}$. The case on which we focus here may be regarded as the simplest one to be analyzed from this viewpoint.

It has long been recognized that, the rational singularity $E_{8}$ being factorial, it contains no smooth curves at all. In fact, a smooth curve is encountered only on those rational surface singularities whose fundamental cycle has a reduced component.

In Section 1, we get a criterion for the existence of smooth curves generically contained in the regular locus of a surface singularity $(S, O)$ of which the above condition is a specialization. This criterion involves the irreducible components of the exceptional fiber of the minimal desingularization of $(S, O)$ over $O$ and a suitable valuative condition, and leads to a decomposi-

1991 Mathematics Subject Classification: 14B05, 14E15, 32B30, 32C45.

Key words and phrases: surface singularities, smooth curves, maximal cycle, wedges.

$\left({ }^{1}\right)$ We became aware of its publication in the special volume "A celebration of John F. Nash Jr." of Duke Math. J. just after finishing writing these pages. 
tion of the set of all such curves into a finite number of mutually disjoint families in one-to-one correspondence with the components just distinguished.

The jets of the parametrizations of the curves in each family coincide up to some order $l$. An equivalent geometric formulation is that these curves go through an infinitely near point $O_{l}$ of $O$ lying on a surface $S_{l}$ obtained from $S$ by a chain of $l$ point blowing-ups. In addition, the strict transform of almost all curves in the family is a smooth branch of a general hypersurface section of $S_{l}$ through $O_{l}$. This is Theorem 1.10 and Remark 1.11.

The application of this result given in Section 2 is in the context of a question concerning the arc structure of surface singularities stated in the introduction of [N]. This question is also reproduced in [G/L2], Problem 3.2, and the connection is explained in Section 3 and [L-J]. Roughly speaking, it means that a smooth curve of one family cannot degenerate to a smooth curve of another one; indeed, a wedge on $(S, O)$ centered at a smooth curve $\Gamma$ (see Definitions 2.1) may be interpreted as a one-parameter deformation of the coefficients of the parametrization of $\Gamma$.

Notation. From now on, $(S, O)$ will denote a surface singularity, i.e. the spectrum of an equicharacteristic complete local ring $A$ of Krull dimension two whose closed point $O$ is singular; furthermore, it will be tacitly assumed that $A$ is reduced and equidimensional, that its residue field $k$ is algebraically closed and that a field of representatives has been fixed. Sing $S$ and $\operatorname{Reg} S$ will denote respectively the singular and the regular locus of $(S, O)$; and $\mathcal{L}$ will be the set of smooth curves $\Gamma$ on $(S, O)$ whose generic point lies on $\operatorname{Reg} S$.

1. Families of smooth curves. Any proper and birational morphism $\pi: X \rightarrow(S, O)$ inducing an isomorphism from $\pi^{-1}(\operatorname{Reg} S)$ to $\operatorname{Reg} S$ gives rise to a map of sets $\Phi_{X}: \mathcal{L} \rightarrow \pi^{-1}(O)$ by sending $\Gamma \in \mathcal{L}$ to the exceptional point of its strict transform $\Gamma_{X}$ on $X$. The exceptional fiber $\pi^{-1}(O)$ has a natural scheme structure given by the inverse image ideal sheaf $\mathfrak{m} \mathcal{O}_{X}$ of the maximal ideal $\mathfrak{m}$ of $\mathcal{O}_{S, O}$. The codimension one component of its underlying cycle, denoted by $Z_{X}$ in the sequel, is the so-called maximal cycle of $\pi$; its support $\left|Z_{X}\right|$ is not empty if and only if $\pi$ is not a finite morphism.

When $X$ is nonsingular, the image of the "fiber map" $\Phi_{X}$ is described through the schematic exceptional fiber as follows.

1.1. Proposition. Let $\pi: X \rightarrow(S, O)$ be a desingularization and let $Q \in \pi^{-1}(O)$.

(i) If $Q$ is isolated in $\pi^{-1}(O)$, then $Q \in \Phi_{X}(\mathcal{L})$ if and only if there exists a regular system of parameters $(u, v)$ of $\mathcal{O}_{X, Q}$ and an integer $m \geq 1$ such that $\mathfrak{m} \mathcal{O}_{X, Q}=\left(u, v^{m}\right)$. 
(ii) If $Q \in\left|Z_{X}\right|$, then $Q \in \Phi_{X}(\mathcal{L})$ if and only if there exists a regular system of parameters $(u, v)$ of $\mathcal{O}_{X, Q}$ such that $\mathfrak{m} \mathcal{O}_{X, Q}=(u)$.

Proof. Let $x$ be the greatest common divisor of the elements in $\mathfrak{m} \mathcal{O}_{X, Q}$ and write $\mathfrak{m} \mathcal{O}_{X, Q}=x I$ for some ideal $I$ in $\mathcal{O}_{X, Q}$. If $\Gamma \in \mathcal{L}$ and $Q=\Phi_{X}(\Gamma)$, a formal parametrization of $\Gamma$ in $(S, O)$ factors through a local homomorphism $\mathcal{O}_{X, Q} \rightarrow k[[t]]$ such that $\operatorname{ord}_{t} \mathfrak{m} \mathcal{O}_{X, Q}=1$, where $\operatorname{ord}_{t}$ denotes the $(t)$-adic valuation in $k[[t]]$.

In case (i), $x$ is a unit and $I$ is primary for the maximal ideal $M$ of $\mathcal{O}_{X, Q}$. So we have $\operatorname{ord}_{t} I=1$ and, a fortiori, $\operatorname{ord}_{Q} I=\max \left\{n \mid I \subset M^{n}\right\}=1$. Any $u \in I \backslash M^{2}$ is part of a regular system of parameters $(u, v)$ of $\mathcal{O}_{X, Q}$ and $I=\left(u, v^{m}\right)$ with $m=\operatorname{ord}_{v} I \mathcal{O}_{X, Q} /(u) \geq 1$.

In case (ii), $x$ is not a unit. So we have

$$
1 \leq \operatorname{ord}_{t} x \leq \operatorname{ord}_{t} x+\operatorname{ord}_{t} I=1 .
$$

Therefore $\operatorname{ord}_{t} x=\operatorname{ord}_{Q} x=1$ and $\operatorname{ord}_{t} I=\operatorname{ord}_{Q} I=0$; the function $x$ is part of a regular system of parameters of $\mathcal{O}_{X, Q}$ and we have $\mathfrak{m} \mathcal{O}_{X, Q}=(x)$.

Conversely, if (i) or (ii) holds, the projection on $(S, O)$ of any formal curve $\widetilde{\Gamma}$ on $(X, Q)$ whose parametrization sends $u$ to $t$ is a smooth curve on $(S, O)$. By imposing the generic point of $\widetilde{\Gamma}$ to lie in $\pi^{-1}(\operatorname{Reg} S)$, we get a curve in $\mathcal{L}$.

This proposition has two immediate consequences, namely a criterion for $\mathcal{L}$ to be nonempty and a natural expression of $\mathcal{L}$ as a disjoint union of finitely many families, joined together in the following statement.

1.2. Corollary. Let $\pi$ be the minimal desingularization of $(S, O)$. For any irreducible component $E$ of $\pi^{-1}(O)$, let $\operatorname{ord}_{E}$ denote the divisorial valuation of the function field of $(S, O)$ given by the filtration of $\mathcal{O}_{X, E}$ by the powers of its maximal ideal. Then

(i) The components $E$ such that $\mathcal{L}_{E}:=\left\{\Gamma \in \mathcal{L} \mid \Phi_{X}(\mathcal{L}) \in E\right\} \neq \emptyset$ are those for which $\operatorname{ord}_{E} \mathfrak{m} \mathcal{O}_{X}=1$.

(ii) The set $\mathcal{L}$ is the disjoint union of the $\mathcal{L}_{E}$.

1.3. This motivates the introduction of some terminology which we will use from now on in this paper.

By a family $\left({ }^{2}\right)$ of smooth curves on $(S, O)$, we will mean any of the nonempty subsets $\mathcal{L}_{E}$ introduced in Corollary 1.2. If $E$ is a point, the family $\mathcal{L}_{E}$ will be said to be small. Each family lies on one sheet (i.e. analytically

$\left({ }^{2}\right)$ This definition does not coincide with the one given by Nash in $[\mathrm{N}]$. In fact, our Theorem 2.3 below is intended to be an intermediate step towards proving that a family of arcs as defined by Nash contains at most one of our families of smooth curves. 
irreducible component) of $(S, O)$. Note that $\mathcal{L}_{E}$ is small if and only if the normalization of the sheet on which it lies is nonsingular.

1.4. It may happen that the general hypersurface section of $(S, O)$ has smooth branches (i.e. analytically irreducible components). A family containing such a branch will be said to be a first order family; before going further in the description of these families, we need to specify what we mean by a general hypersurface section. This will be done in terms of the normalized blowing-up of $(S, O)$ with center $O$, that is, the composition $\bar{\sigma}_{1}=\sigma_{1} \circ n_{1}$ of the blowing-up $\sigma_{1}: S_{1} \rightarrow(S, O)$ of $O$ and the normalization $n_{1}: \bar{S}_{1} \rightarrow S_{1}$. For simplicity, $Z_{1}$ (resp. $\bar{Z}_{1}$ ) will denote the maximal cycle of $\sigma_{1}$ (resp. $\bar{\sigma}_{1}$ ) instead of $Z_{S_{1}}$ (resp. $Z_{\bar{S}_{1}}$ ); and $C_{S, O}$ (resp. $T_{S, O}$ ) will denote the tangent cone (resp. the Zariski tangent space) of $S$ at $O$, as usual.

Recall that a hypersurface section of $(S, O)$ is a "curve", i.e. a Cartier divisor on $(S, O)$ given by a local equation $h=0$ for some $h \in \mathfrak{m}$ which is not a zero divisor in $\mathcal{O}_{S, O}$. Here we will say that it is general if $h \notin \mathfrak{m}^{2}$ and if the hyperplane $H$ in Proj $T_{S, O}$ given by $h \bmod \mathfrak{m}^{2}=0$ intersects the curve $\operatorname{Proj}\left|C_{S, O}\right|=\operatorname{Proj}\left|Z_{1}\right|$ transversally at regular points of $\left|Z_{1}\right|$ onto which neither singular points of $\bar{S}_{1}$ nor branch points of $\left|\bar{Z}_{1}\right| \rightarrow\left|Z_{1}\right|$ project and other than the exceptional points of the strict transform of $\operatorname{Sing} S$ (if $O$ is not a isolated singular point) ([G-S]).

In the sequel, the lines on $C_{S, O}$ corresponding to the above "prohibited" points of $\left|Z_{1}\right|$ will be said to be special.

In view of Bertini's theorem, the set of hyperplanes in $\mathbb{P} T:=\operatorname{Proj} T_{S, O}$ with the properties just listed forms a Zariski open dense subset of the linear system $\mathcal{O}_{\mathbb{P} T}(1)$

General hypersurface sections of $(S, O)$ need not be analytically isomorphic. But they have in common the following "equisingularity" properties which will be enough for our purpose: any of them is generically reduced, is reduced if and only if $\mathcal{O}_{S, O}$ is Cohen Macaulay and has $-\left(\bar{Z}_{1} \cdot\left|\bar{Z}_{1}\right|\right)$ branches, each irreducible component $\bar{F}$ of $\left|\bar{Z}_{1}\right|$ contributing to $-\left(\bar{Z}_{1} \cdot \bar{F}\right)>0$ branches whose strict transforms on $\bar{S}_{1}$ meet $\bar{F}$ transversally and whose multiplicity at $O$ is the multiplicity $m_{\bar{F}}$ of $\bar{F}$ in the maximal cycle $\bar{Z}_{1}$. A component $\bar{F}$ such that $m_{\bar{F}}=1$ will be said to be a reduced component of $\bar{Z}_{1}$.

First order families of smooth curves are identified from their images by the fiber map $\Phi_{\bar{S}_{1}}$ as follows.

1.5. Proposition. Let $\mathcal{L}_{E}$ be a family of smooth curves. If $\mathcal{L}_{E}$ is a first order family, then there exists a reduced component $\bar{F}_{1}$ of $\bar{Z}_{1}$ such that $\Phi_{\bar{S}_{1}}\left(\mathcal{L}_{E}\right)=\bar{F}_{1} \cap \operatorname{Reg} \bar{S}_{1} \cap \operatorname{Reg}\left|\bar{Z}_{1}\right|$. If not, then there exists a singular point $\bar{O}_{1}$ of $\bar{S}_{1}$ such that $\Phi_{\bar{S}_{1}}\left(\mathcal{L}_{E}\right)=\bar{O}_{1}$. 
Pr o of. Let $\bar{\pi}_{1}: X_{1} \rightarrow \bar{S}_{1}$ be the minimal desingularization of $\bar{S}_{1}$. The morphism $\tau_{1}: X_{1} \rightarrow X$ factoring $\bar{\sigma}_{1} \circ \bar{\pi}_{1}$ is the composition of the sequence of point blowing-ups with minimal length such that $\mathfrak{m} \mathcal{O}_{X_{1}}$ is invertible. It follows from Proposition 1.1 that $\Phi_{X_{1}}\left(\mathcal{L}_{E}\right)$ is contained in a single reduced component $E_{1}$ of $Z_{X_{1}}$, namely the strict transform of $E$ if $E$ is a curve, and that of the exceptional curve created by blowing up $E$ if $E$ is a point. Indeed, if $\operatorname{dim} E=1, \mathfrak{m} \mathcal{O}_{X}$ is invertible at any $Q \in \Phi_{X}\left(\mathcal{L}_{E}\right)$ and if $\operatorname{dim} E=0$ and $\mathfrak{m} \mathcal{O}_{X, E}=\left(u, v^{m}\right)$, it is easily checked that $m$ point blowing-ups are necessary to make the total transform of $\mathfrak{m} \mathcal{O}_{X}$ invertible over a neighborhood of $E$ and that $E_{1}$ is the unique reduced component of $Z_{X_{1}}$ contracted to $E$.

Now, in view of $1.4, \mathcal{L}_{E}$ is a first order family if and only if the image of $E_{1}$ on $\bar{S}_{1}$ is a curve. This is because the exceptional points of the strict transform on $\bar{S}_{1}$ of a general hypersurface section are regular points of $\bar{S}_{1}$ and that $E_{1}$ being a reduced component of $Z_{X_{1}}$, either $\bar{\pi}_{1}\left(E_{1}\right)$ is a reduced component $\bar{F}_{1}$ of $Z_{\bar{S}_{1}}$, or the minimal desingularization $\bar{\pi}_{1}$ of $\bar{S}_{1}$ contracts $E_{1}$ to a singular point $\bar{O}_{1}$ of $\bar{S}_{1}$.

In the first case, $\Phi_{X_{1}}\left(\mathcal{L}_{E}\right)=E_{1} \cap \operatorname{Reg}\left|Z_{X_{1}}\right|$ by $1.1(\mathrm{ii})$, so $\bar{\pi}_{1}$ is an isomorphism on a neighborhood of $\Phi_{X_{1}}\left(\mathcal{L}_{E}\right)$ and $\Phi_{\bar{S}_{1}}\left(\mathcal{L}_{E}\right)=\bar{\pi}_{1}\left(\Phi_{X_{1}}\left(\mathcal{L}_{E}\right)\right)=$ $\bar{F}_{1} \cap \operatorname{Reg} \bar{S}_{1} \cap \operatorname{Reg}\left|\bar{Z}_{1}\right|$.

A small family of smooth curves may be a first order family as well. A first corollary of Proposition 1.5 is that the families enjoying both properties are in one-to-one correspondence with the nonsingular sheets of $S$ at $O$.

1.6. Corollary. A small family of smooth curves is a first order family if and only if $O$ is a nonsingular point of the sheet of $S$ on which it lies. Conversely, any nonsingular sheet of $S$ at $O$ carries such a family.

Pro of. While proving Proposition 1.5, we have shown that the family $\mathcal{L}_{E}$ is a first order family if and only if the image on $\bar{S}_{1}$ of the reduced component $E_{1}$ of $Z_{X_{1}}$ containing $\Phi_{X_{1}}\left(\mathcal{L}_{E}\right)$ is a curve $\bar{F}_{1}$.

Now by the projection formula, $\left(Z_{X_{1}} \cdot E_{1}\right)=\left(\bar{Z}_{1} \cdot \bar{F}_{1}\right) \neq 0$ if this happens (since, up to sign, it coincides with the number of branches of the general hypersurface section whose strict transforms meet $\left.\bar{F}_{1}\right)$ and is 0 if $\bar{\pi}_{1}\left(E_{1}\right)$ is a point.

For a small family such that $\mathfrak{m} \mathcal{O}_{X, E}=\left(u, v^{m}\right)$, the intersection number $\left(Z_{X_{1}} \cdot E_{1}\right)$ does not vanish if and only if $m=1$. Indeed, the intersection matrix of the components of $\left|Z_{X_{1}}\right|$ which project to $E$ is read off the weighted dual graph

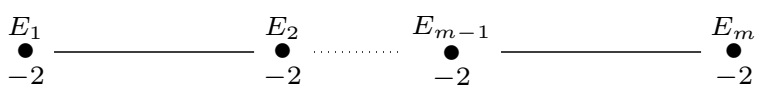

$E_{i}$ being the strict transform of the exceptional curve created by the $i$ th blowing-up and one has $Z_{X_{1}} \equiv E_{1}+2 E_{2}+\ldots+m E_{m}$ up to curves which do not intersect $E_{1}$. 
So, if $\mathcal{L}_{E}$ is both a first order family and small and if it lies on the sheet $\mathcal{S}$ of $S, \mathcal{O}_{X, E}$ is a free module of rank 1 over $\mathcal{O}_{\mathcal{S}, O}$. Therefore $\mathcal{O}_{X, E}$ and $\mathcal{O}_{\mathcal{S}, O}$ coincide and $\mathcal{S}$ is regular at $O$.

The converse is clear.

Another corollary of Proposition 1.5 is a characterization of the first order families in terms of $\bar{Z}_{1}$. More precisely, we have:

1.7. Corollary. The map $\Phi_{\bar{S}_{1}}$ induces a one-to-one correspondence between first order families of smooth curves and reduced components of $\bar{Z}_{1}$. A reduced component of $\bar{Z}_{1}$ comes from a small family if and only if it is a nonsingular rational curve lying on $\operatorname{Reg} \bar{S}_{1}$ with self-intersection -1 .

Proof. The second part of the assertion follows from the fact that, if $\mathcal{L}_{E}$ is small, then the morphism $\tau_{1}: X_{1} \rightarrow X$ coincides with the blowing-up of $E$ over a neighborhood of $E$, so the restriction of $\bar{\pi}_{1}$ to a neighborhood of $\bar{F}_{1}$ is an isomorphism.

1.8. Therefore, depending on whether the family $\mathcal{L}_{E}$ is a first order family or not, the set $T_{E}$ of tangent lines to $\Gamma \in \mathcal{L}_{E}$ consists of all but possibly finitely many special lines through $O$ on an irreducible component of $C_{S, O}$ or of a single special line of this tangent cone.

Note that in the first case, $T_{E}$ may contain special lines of $C_{S, O}$. In the last case, let $O_{1} \in S_{1}$ be the common tangent direction to every $\Gamma \in \mathcal{L}_{E}$ and let $E_{1}$ be the irreducible exceptional curve on the minimal desingularization $X_{1}$ of $\bar{S}_{1}$ (or $S_{1}$ ) containing $\Phi_{X_{1}}\left(\mathcal{L}_{E}\right)$; according to $1.3, E_{1}$ gives rise to a family $\mathcal{L}_{1}$ of smooth curves on $\left(S_{1}, O_{1}\right)$ which contains the strict transform of every $\Gamma \in \mathcal{L}_{E}$. If $\mathcal{L}_{1}$ is not a first order family, the strict transform $E_{2}$ of $E_{1}$ on the minimal desingularization $X_{2}$ of the surface $S_{2}$ obtained by blowing up $O_{1}$ in $S_{1}$ is contracted to a point $O_{2} \in S_{2}$ which is the common tangent direction to every $\Gamma \in \mathcal{L}_{1}$ and corresponds to a family $\mathcal{L}_{2}$ of smooth curves on $\left(S_{2}, O_{2}\right)$ which contains the strict transform on $S_{2}$ of every $\Gamma \in \mathcal{L}_{1}$, hence of every $\Gamma \in \mathcal{L}_{E}$. And so on... so long as a first order family $\mathcal{L}_{i}$ does not show up. Note that none of the $\mathcal{L}_{i}, i \geq 1$, is small. This leads to the following definition and "dévissage" of $\mathcal{L}_{E}$.

1.9. Definition. A chain of infinitely near points of $O$ on $(S, O)$ (i.e. a sequence (finite or infinite) of points $\left\{O_{0}=O, O_{1}, \ldots, O_{i}, \ldots\right\}$ such that for each $i>0, O_{i}$ is mapped to $O_{i-1}$ by the blowing-up $\sigma_{i}: S_{i} \rightarrow S_{i-1}$ of $O_{i-1}$ and $S_{0}=S$ ) will be said to be special if for each $i>0, O_{i}$ is the direction of a special line on $C_{S_{i-1}, O_{i-1}}$.

1.10. TheOREM. Let $\mathcal{L}_{E}$ be a family of smooth curves. There exists a finite special chain $\left\{O_{i}\right\}_{0 \leq i \leq l}$ of infinitely near points of $O$ on $(S, O)$ and a reduced component $\bar{F}_{l+1}$ of the maximal cycle $\bar{Z}_{l+1}$ of $\sigma_{1} \circ \ldots \circ \bar{\sigma}_{l+1}$, $\bar{\sigma}_{l+1}: \bar{S}_{l+1} \rightarrow S_{l}$ being the normalized blowing-up of $O_{l}$, such that: 
(i) $\Phi_{S_{i}}\left(\mathcal{L}_{E}\right)=O_{i}, 1 \leq i \leq l$,

(ii) $\Phi_{\bar{S}_{l+1}}\left(\mathcal{L}_{E}\right)=\bar{F}_{l+1} \cap \operatorname{Reg} \bar{S}_{l+1} \cap \operatorname{Reg}\left|\bar{Z}_{l+1}\right|$.

In addition, if $\mathcal{L}_{E}$ is not small, the birational map $\pi^{-1} \circ \sigma_{1} \circ \ldots \circ \bar{\sigma}_{l+1}$ identifies neighborhoods of $\Phi_{\bar{S}_{l+1}}\left(\mathcal{L}_{E}\right)$ and $\Phi_{X}\left(\mathcal{L}_{E}\right)$.

Proof. Pick a curve $\Gamma$ in $\mathcal{L}_{E}$ and let $\left\{O_{i}\right\}_{i \in \mathbb{N}}$ be the chain of infinitely near points of $O$ lying on $\Gamma$. Since $\Gamma$ is smooth and generically contained in Reg $S$, there exists an integer $N$ such that $O_{N} \in \operatorname{Reg} S_{N}$ ([L], Proposition 1.28, [L/T], Chap. II, Theorem 2.13). So $C_{S_{N}, O_{N}}$ carries no special lines at all and the infinite chain $\left\{O_{i}\right\}_{i \in \mathbb{N}}$ may not be special.

Therefore the smallest $i$ such that the family $\mathcal{L}_{i}$ of 1.8 is a first order family (or equivalently the strict transform $E_{i+1}$ of $E_{1}$ on the minimal desingularization $X_{i+1}$ of $S_{i+1}$ is not contracted to a point on $S_{i+1}$ ) is an integer $l \geq 0$. As a consequence of Proposition 1.5, the chain $\left\{O_{i}\right\}_{0 \leq i \leq l}$ is special and (i) holds.

Let us now prove (ii). The minimal desingularizations $\pi_{i}: X_{i} \rightarrow S_{i}$, $1 \leq i \leq l$, and $\bar{\pi}_{l+1}: X_{l+1} \rightarrow \bar{S}_{l+1}$ are the vertical arrows of a sequence of commutative diagrams:

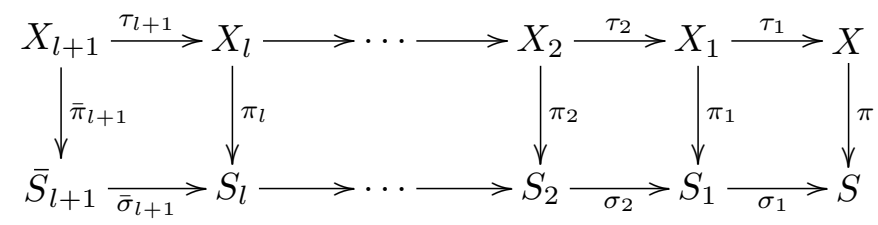

where $\tau_{i+1}$ is the sequence of point blowing-ups with minimal length making the inverse image ideal sheaf $\mathfrak{m}_{i} \mathcal{O}_{X_{i}}$ of the maximal ideal $\mathfrak{m}_{i}$ of $\mathcal{O}_{S_{i}, O_{i}}$ invertible, $0 \leq i \leq l$.

Applying 1.1(ii) to $\pi_{i}$, we see that $\mathfrak{m}_{i} \mathcal{O}_{X_{i}}$ is invertible at any $Q \in \Phi_{X_{i}}\left(\mathcal{L}_{i}\right)$ for $1 \leq i \leq l$. Since $\Phi_{X_{i}}\left(\mathcal{L}_{E}\right) \subset \Phi_{X_{i}}\left(\mathcal{L}_{i}\right)$, the strict transform $E_{l+1}$ of $E_{1}$ is the only irreducible component of the support of the maximal cycle $Z_{X_{l+1}}$ of $\pi \circ \tau_{1} \circ \ldots \circ \tau_{l+1}$ containing $\Phi_{X_{l+1}}\left(\mathcal{L}_{E}\right)$ and moreover $\tau_{l+1} \circ \ldots \circ \tau_{2}$ is an isomorphism on a neighborhood of $\Phi_{X_{l+1}}\left(\mathcal{L}_{E}\right)$. Now $E_{1}$ being a reduced component of the maximal cycle $Z_{X_{1}}$ of $\pi \circ \tau_{1}, E_{l+1}$ is a reduced component of $Z_{X_{l+1}}$. In addition, applying 1.1(ii) to $\pi \circ \tau_{1} \circ \ldots \circ \tau_{l+1}$, we find that $\Phi_{X_{l+1}}\left(\mathcal{L}_{E}\right)=E_{l+1} \cap \operatorname{Reg}\left|Z_{l+1}\right|$.

But the image $\bar{F}_{l+1}$ of $E_{l+1}$ on $\bar{S}_{l+1}$ being a curve, this equality forces $\bar{\pi}_{l+1}$ to be an isomorphism on a neighborhood of $\Phi_{X_{l+1}}\left(\mathcal{L}_{E}\right)$. Consequently, $\bar{F}_{l+1}$ is a reduced component of the maximal cycle $\bar{Z}_{l+1}$ of $\sigma_{1} \circ \ldots \circ \bar{\sigma}_{l+1}$ and (ii) holds.

To complete the proof of the theorem, it is enough to observe that $\tau_{1}$ is an isomorphism at any $Q \in \Phi_{X_{1}}\left(\mathcal{L}_{E}\right)$ if $\mathcal{L}_{E}$ is not small. 
1.11. Remark. Since the first order family $\mathcal{L}_{l}$ on $\left(S_{l}, O_{l}\right)$ contains the strict transform of every $\Gamma \in \mathcal{L}_{E}$, the description of $\Phi_{\bar{S}_{l+1}}\left(\mathcal{L}_{E}\right)$ inside $\bar{F}_{l+1}$ given above combined with that of $\Phi_{\bar{S}_{l+1}}\left(\mathcal{L}_{l}\right)$ given in Proposition 1.5 implies that $\bar{F}_{l+1}$ is also a reduced component of the maximal cycle of $\bar{\sigma}_{l+1}$. Moreover, it shows that the strict transform on $S_{l}$ of almost all $\Gamma \in \mathcal{L}_{E}$ is a smooth branch of a general hypersurface section of $\left(S_{l}, O_{l}\right)$. We will say that the family $\mathcal{L}_{E}$ has order $l+1$.

In view of the universal property of normalization, any chain $\mathcal{C}=$ $\left\{O_{i}\right\}_{0 \leq i \leq l}$ of infinitely near points of $O$ on $(S, O)$ yields a commutative diagram:

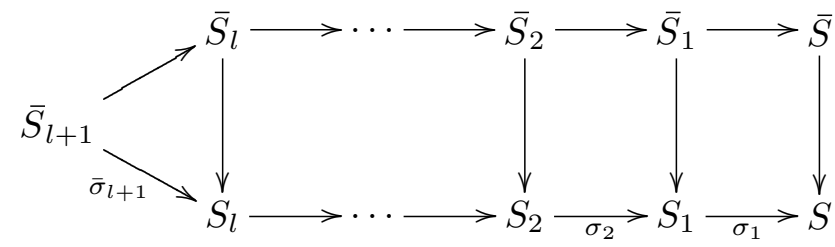

where $\sigma_{i}$ is the blowing-up of $O_{i-1}, 1 \leq i \leq l, \bar{\sigma}_{l+1}$ is the normalized blowing-up of $O_{l}$ and the vertical arrows are normalizations. More precisely, $\mathfrak{m}_{i}$ being the maximal ideal of $\mathcal{O}_{S_{i}, O_{i}}$, the map from $\bar{S}_{i+1}$ to $\bar{S}_{i}$ is the normalized blowing-up of $\mathfrak{m}_{i} \mathcal{O}_{\bar{S}_{i}}$.

We have the following characterization of the families of order $l+1$ going through a given special chain with $l+1$ points in terms of the maximal cycle $Z_{\sigma_{1} \circ \ldots \circ \bar{\sigma}_{l+1}}$, parallel to Corollary 1.7.

1.12. Corollary. Given a special chain $\mathcal{C}=\left\{O_{i}\right\}_{0 \leq i \leq l}$ of infinitely near points of $O$ on $(S, O)$, the fiber map $\Phi_{\bar{S}_{l+1}}$ induces a one-to-one correspondence between families of smooth curves of order $l+1$ going through the points of $\mathcal{C}$ and reduced components of the maximal cycle $\bar{Z}_{l+1}$ of $\sigma_{1} \circ$ $\ldots \circ \bar{\sigma}_{l+1}$ contracted to a singular point $\bar{O}_{i}$ of $\bar{S}_{i}$ above $O_{i}, 1 \leq i \leq l$. Such a component comes from a small family if and only if $\bar{F}_{l+1}$ is contracted to a regular point $\bar{O}$ of $\bar{S}$.

Pr o of. Let $X_{i}$ (resp. $X_{0}=X$ ) be the minimal desingularization of $\bar{S}_{i}$, $1 \leq i \leq l+1\left(\operatorname{resp} . \bar{S}_{0}:=\bar{S}\right)$.

The reduced component $\bar{F}_{l+1}$ of $\bar{Z}_{l+1}$ associated with the family $\mathcal{L}_{E}$ in Theorem 1.10 has the required property since the Zariski closure of its image on $X_{i}$ (resp. $\left.X\right)$ is a curve $E_{i}$ contracted to $O_{i}$ for $1 \leq i \leq l$ (resp. $E$ ).

Conversely, consider a reduced component $\bar{F}_{l+1}$ of $\bar{Z}_{l+1}$ contracted to a point $\bar{O}_{l} \in \bar{S}_{l}$ above $O_{l}$ and let $E_{i}$ be the Zariski closure of its image on $X_{i}, 0 \leq i \leq l+1$. Now pick a curve $\Gamma_{0} \in \mathcal{L}$ whose strict transform on $X_{l+1}$ intersects $E_{l+1}$ and no other exceptional curve of $X_{l+1}$ over $O$. Such a curve exists by Proposition 1.1(ii). For any $i, 0 \leq i \leq l$, the strict transform $\Gamma_{i}$ of 
$\Gamma_{0}$ on $S_{i}$ is smooth, generically contained in $\operatorname{Reg} S_{i}$ and it goes through $O_{i}$. The exceptional point of its strict transform on $X_{i}, \Phi_{X_{i}}\left(\Gamma_{i}\right)$, lies on $E_{i}$ and projects to the image $\bar{O}_{i}$ of $\bar{O}_{l}$ on $\bar{S}_{i}$.

Now observe that for any such $i$, if $\bar{O}_{i} \in \operatorname{Sing} \bar{S}_{i}$ and $E_{i}$ is a point, then $E_{i+1}$ is also a point. Indeed, if so, then $E_{i}$ is not an isolated point of the exceptional fiber of $X_{i} \rightarrow S_{i}$ over $O_{i}$ and by Proposition 1.1(ii) applied to $\left(S_{i}, O_{i}\right)$ and $E_{i}=\Phi_{X_{i}}\left(\Gamma_{i}\right)$, the morphism $\tau_{i+1}: X_{i+1} \rightarrow X_{i}$ does not factor through the blowing-up of $E_{i}$; hence $E_{i+1}$ may not be a curve.

Therefore, $E_{l+1}$ being a curve, if we assume that $\bar{O}_{i} \in \operatorname{Sing} \bar{S}_{i}, 1 \leq i \leq l$, then $E_{1}$ is a curve and $E_{0}$ is a curve or a point depending on whether $\bar{O} \in \operatorname{Sing} \bar{S}$ or not. In any case, $E_{0}$ is an irreducible component of the exceptional fiber of $X \rightarrow S$ over $O$, which gives rise to a family $\mathcal{L}_{E_{0}}$ of smooth curves containing $\Gamma_{0}$.

Now if $k+1$ denotes its order, we may not have $k+1<l+1$ since Theorem 1.10(ii) would imply that $\bar{O}_{k+1} \in \operatorname{Reg} \bar{S}_{k+1}$, but by assumption this is a singular point. We may not have $l+1<k+1$ either since by the direct analysis just settled, we would have $\bar{O}_{l+1} \in \operatorname{Sing} \bar{S}_{l+1}$ but this is a regular point by construction. Therefore $k=l$ and the given chain $\mathcal{C}$ is the one associated with $\mathcal{L}_{E_{0}}$. Finally, since no point of $\Phi_{\bar{S}_{l+1}}\left(\mathcal{L}_{E_{0}}\right)$ lies on two distinct irreducible components of $\left|\bar{Z}_{l+1}\right|$ and $\Phi_{\bar{S}_{l+1}}\left(\Gamma_{0}\right) \in \bar{F}_{l+1}$, the reduced component of $\left|\bar{Z}_{l+1}\right|$ attached to $\mathcal{L}_{E_{0}}$ is $\bar{F}_{l+1}$.

We close this section by one remark and some examples.

1.13. Any chain (finite or infinite) of infinitely near points of $O$ on $(S, O)$ may also be regarded as a chain of infinitely near points of $O$ on a formal nonsingular space $(Z, O)$ containing $(S, O)$. The points in the chain lie on a smooth formal curve on $(Z, O)$ if and only if any $Q \in \mathcal{C}$ distinct from $O$ is proximate to exactly one point in $\mathcal{C}$, its antecedent. Recall that according to Enriques' terminology, $Q$ is said to be proximate to $P$ if $Q$ is infinitely near $P$ and lies on the strict transform of the exceptional divisor created by blowing up $P$. We will say that such a chain is regular.

If $(S, O)$ has an isolated singularity at $O$, any chain of infinitely near points of $O$ on $(S, O)$ which is both special and regular is finite, once again by $[\mathrm{L} / \mathrm{T}]$ or $[\mathrm{L}]$, hence there exist only finitely many such chains.

1.14. The families of smooth curves on a normal surface singularity are in one-to-one correspondence with the reduced components of the maximal cycle of its minimal desingularization $\pi$ by 1.2. For a rational surface singularity, the maximal cycle of $\pi$ and the fundamental cycle of its weighted dual graph $\Gamma$ coincide $[\mathrm{A}]$. Among rational surface singularities, we have three increasingly restrictive conditions:

sandwiched $\nsupseteq$ minimal $\nsupseteq$ cyclic quotients 
depending only on $\Gamma$; see $[\mathrm{S}]$. Minimal ones are those having a reduced fundamental cycle.

The fundamental cycle of a sandwiched singularity has at least one reduced component. Indeed, there exists a nonsingular graph $\Gamma^{*}$ containing $\Gamma$ such that the curves represented by vertices in $\Gamma^{*} \backslash \Gamma$ are exactly those with self-intersection $-1 ; \Gamma^{*}$ is the weighted dual graph of a configuration of curves which blow down to a nonsingular point $O$. Blowing up $O$ creates a component represented by a vertex in $\Gamma$ and having multiplicity one in the fundamental cycle $Z^{*}$ of $\Gamma^{*}$. The minimality property of the fundamental cycle $Z$ of $\Gamma$ implies that the cycle obtained from $Z^{*}$ by deleting the $(-1)$ curves is greater than or equal to $Z$.

1.15. Corollary. Any sandwiched singularity contains a smooth curve.

The rational double points $D_{n}, E_{n}$ are not sandwiched. By inspecting their fundamental cycles, one finds respectively 3, 2, 1 families of smooth curves on $D_{n}, E_{6}, E_{7}$.

Among nonrational singularities, the complete intersection defined by $d-2$ general elements of a finitely supported ideal in $\mathbb{C}\left[\left[X_{1}, \ldots, X_{d}\right]\right]$ provides an example of a surface singularity whose general hypersurface section is a union of smooth branches.

A small family of smooth curves appears on the germ at the origin of the Whitney umbrella $x^{2}-y^{2} z=0$ since its normalization is given by $x=u v$, $y=v, z=v^{2}$.

\section{Wedges centered at a smooth curve}

2.1. Following freely a classical terminology (compare for example with $[\mathrm{W}]$, I.5) we will say that a local continuous morphism $\varphi$ from $\mathcal{O}_{S, O}$ to a formal power series ring $R$ in two variables with coefficients in the residue field $k$ of $S$ at $O$ is a wedge on $(S, O)$ if the kernel of $\varphi$ is a minimal prime ideal of $\mathcal{O}_{S, O}$. This is also equivalent to saying that the image of the associated morphism $\left(B_{2}, 0\right):=\operatorname{spec} R \rightarrow(S, O)$ is Zariski dense in some sheet (or analytically irreducible component) of $(S, O)$.

We will say that the wedge $\varphi$ is centered at a parametrized curve on $(S, O)$ given by $h: \mathcal{O}_{S, O} \rightarrow k[[t]]$ if $h$ factors through $\varphi$, that is, if it can be lifted to $B_{2}$.

This section is aimed at proving that a morphism $\left(B_{2}, 0\right) \rightarrow(S, O)$ given by a wedge centered at a curve in $\mathcal{L}$ factors through the minimal desingularization of $(S, O)$. This will be an easy consequence of the analysis in Section 1 and of the following observation.

2.2. Proposition. Let $p:\left(B_{2}, 0\right) \rightarrow(S, O)$ be given by a wedge centered at a curve $\Gamma$ in $\mathcal{L}$. 
(i) If $p^{-1}(O)$ is a curve, then $p$ factors through the blowing-up of $O$.

(ii) If $p^{-1}(O)=0$, then the normalization of the sheet of $(S, O)$ on which $\Gamma$ lies is nonsingular.

P r o of. The argument already used to prove Proposition 1.1(ii) remains valid if $\varphi^{-1}(O)$ is a curve. So, $\mathfrak{m}$ denoting the maximal ideal of $\mathcal{O}_{S, O}, \mathfrak{m} R$ is generated by one of the elements in a regular system of parameters of $R$, hence (i).

Assume now that $p^{-1}(O)=0$ and let $\bar{S}$ denote the normalization of $S$. The curve $\Gamma$ being smooth and generically contained in $\operatorname{Reg} S$, the same holds for its strict transform $\bar{\Gamma}$ on $\bar{S}$. Now by the universal property of normalization, the wedge defining $p$ factors through the local ring $B$ of $\bar{S}$ at the exceptional point $Q$ of $\bar{\Gamma}$, giving rise to an injective wedge $\bar{\varphi}: B \rightarrow R$ on $(\bar{S}, Q)$ which is centered at $\bar{\Gamma}$. The local ring $\mathcal{O}_{S, O}$ being complete by hypothesis, $B$ is a complete local domain, hence $R$ is a finite $B$-module. So the morphism $\bar{p}:\left(B_{2}, 0\right) \rightarrow(\bar{S}, Q)$ given by $\bar{\varphi}$ is finite and surjective and $\bar{p}$ induces an isomorphism from a smooth curve $\Delta$ on $\left(B_{2}, 0\right)$ to $\bar{\Gamma}$.

The ring $R$ being factorial, $\Delta$ is a principal divisor on $\left(B_{2}, 0\right)$. Here as in the algebraic context, the rational equivalence of cycles pushes forward; the induced morphism $\bar{p}_{\mid \Delta}: \Delta \rightarrow \bar{\Gamma}$ being unramified, $\bar{\Gamma}$ is also a principal divisor. In fact, $K$ being the fraction field of $B, R \otimes_{B} K$ is a finite-dimensional vector space over $K$ and if $\Delta=\operatorname{div} s$, one can check that $\bar{\Gamma}=\operatorname{div} N(s)$ where $N(s)$ is the determinant of the $K$-linear endomorphism of $R \otimes_{B} K$ given by multiplication by $s \otimes 1$. As a consequence, the multiplicity of $\bar{\Gamma}$, which is one by assumption, is not smaller than the multiplicity of $B$. Therefore $B$ is regular. In other words, the normalization of the sheet of $(S, O)$ on which $\Gamma$ lies is nonsingular.

Observe that in case (ii), the family of smooth curves containing $\Gamma$ is small. We are now ready to prove:

2.3. THEOREM. If a morphism $p:\left(B_{2}, 0\right) \rightarrow(S, O)$ is given by a wedge centered at a smooth curve $\Gamma$ whose generic point lies in $\operatorname{Reg} S$, then $p$ factors through the minimal desingularization $X$ of $(S, O)$.

P r o of. Let $\mathcal{L}_{E}$ be the family of smooth curves on $(S, O)$ which contains $\Gamma$. If $\mathcal{L}_{E}$ is small, the claim follows immediately from the universal property of normalization, since the normalization of the sheet containing $\mathcal{L}_{E}$ is nonsingular. If not, let $l+1 \geq 1$ be the order of the family $\mathcal{L}_{E}$; if $Q:=\Phi_{X}(\Gamma)$, then Theorem 1.10 asserts that the morphism $\mathcal{O}_{S, O} \rightarrow \mathcal{O}_{X, Q}$ coincides (up to $\mathcal{O}_{S, O}$-isomorphism) with the composed morphism

$$
\mathcal{O}_{S, O} \rightarrow \mathcal{O}_{S_{1}, O_{1}} \rightarrow \ldots \rightarrow \mathcal{O}_{S_{l}, O_{l}} \rightarrow \mathcal{O}_{\bar{S}_{l+1}, P}
$$

where $\left\{O_{i}\right\}_{0 \leq i \leq l}$ is the finite special chain of infinitely near points of $O$ provided by $\mathcal{L}_{E}, \sigma_{i}: S_{i} \rightarrow S_{i-1}, 1 \leq i \leq l$, is the blowing-up of $O_{i}, \bar{\sigma}_{l+1}$ is 
the normalized blowing-up of $O_{l}$ and $P:=\Phi_{\sigma_{1} \circ \ldots \circ \bar{\sigma}_{l+1}}(\Gamma)$. Here the claim follows from Proposition 2.2 and once again from the universal property of normalization, since $\mathcal{L}_{E}$ is not small and for any $i, 1 \leq i \leq l$, the family $\mathcal{L}_{i}$ of smooth curves on $\left(S_{i}, O_{i}\right)$ containing the strict transform of $\Gamma$ on $S_{i}$ is not small either by 1.8 .

\section{References}

[A] M. Artin, On isolated rational singularities of surfaces, Amer. J. Math. 88 (1966), 129-136.

[E] D. Eisenbud, Open problems in computational algebraic geometry, in: Computational Algebraic Geometry and Commutative Algebra, D. Eisenbud and L. Robbiano (eds.), Cambridge Univ. Press, 1993, 49-70.

[G-S] G. Gonzalez-Sprinberg, Cycle maximal et invariant d'Euler local des singularités isolées de surface, Topology 21 (1982), 401-408.

[G/L1] G. Gonzalez-Sprinberg et M. Lejeune-Jalabert, Courbes lisses sur les singularités de surface, C. R. Acad. Sci. Paris Sér. I Math. 318 (1994), 653-656.

[G/L2] - - - Sur l'espace des courbes tracées sur une singularité, in: Algebraic Geometry and Singularities, A. Campillo and L. Narváez (eds.), Progr. Math. 134, Birkhäuser, 1996, 9-32.

[L] J. Lipman, Desingularization of two-dimensional schemes, Ann. of Math. 107 (1978), 151-207.

[L-J] M. Lejeune-Jalabert, Arcs analytiques et résolution minimale des singularités des surfaces quasi-homogènes, in: Lecture Notes in Math. 777, Springer, 1980, 303-336.

$[\mathrm{L} / \mathrm{T}] \quad \mathrm{M}$. Lejeune-Jalabert et B. Teissier, Contributions à l'étude des singularités du point de vue du polygone de Newton, Thèse, Université Paris 7, 1973.

[N] J. Nash, Arc structure of singularities, preprint, 1968; Duke Math. J. 81 (1995), $31-38$.

[S] M. Spivakovsky, Sandwiched singularities and desingularization of surfaces by normalized Nash transformations, Ann. of Math. 131 (1990), 411-491.

[W] R. Walker, Reduction of the singularities of an algebraic surface, ibid. 36 (1935), 336-365.

Université de Grenoble I

Institut Fourier

UMR 5582 du CNRS et de l'UJF

UFR de Mathématiques

B.P. 74

38402 St. Martin d'Hères Cedex (France)

E-mail: gonsprin@fourier.ujf-grenoble.fr lejeune@fourier.ujf-grenoble.fr 